\title{
Delay-dependent stability criteria for impulsive high-order Hopfield-Type neural networks with time-varying delays
}

\author{
Hui Yang ${ }^{\text {}}$, Xiaofeng Li and Dongming Wang \\ Shandong Provincial Key Laboratory of Ocean Environment Monitoring Technology \\ aquingbaihui@sina.com,
}

Keywords: Delay-dependent stability, Impulsive, high-order Hopfield-type neural network.

\begin{abstract}
This paper is concerned with the stability analysis for impulsive stochastic high-order Hopfield-type neural network with time-varying delay. Utilizing the Lyapunov-Krasovskii Functional, some new conditions for ensuring asymptotically stability of the neural network are devised. Numerical examples show that the results are effectiveness.
\end{abstract}

\section{Introduction}

In the past two decades, Hopfield neural network (HNN) has been extensively studied, and successfully applied in many areas such as associate memory, pattern recognition and combinatorial optimization in [1-3]. In particular, stability analysis problem is one of the main properties of Hopfield neural network, which is a crucial feature in the design of neural network. The existing results for the above researches with time-delay can be classified into two categories: delay-independent stability and delay-dependent stability.

As is well known now, the stability analysis issues of neural networks in the presence of stochastic perturbations have stirred the research attention. Motivated by these, in this paper we address the problem of delay-dependent stability for high-order impulsive Hopfield neural networks with time-varying delay in the presence of stochastic perturbations. By using a new Lyapunov-Krasovskii functional and Linear Matrix Inequality (LMI), several delay-dependent conditions are presented such that the neural network is asymptotically stable.

The rest of this paper is organized as follows: in section 2, we give formulation and preliminaries for an impulsive high-order Hopfield neural network with time-varying delays; in section 3, some sufficient conditions are presented for the asymptotical stability of the system; in section 4 , we give the conclusions.

\section{Problem formulation}

Consider the impulsive high-order Hopfield-type neural networks with time-varying delays described by

$$
\begin{aligned}
& C_{i} \frac{d x_{i}(t)}{d_{t}}=-\frac{x_{i}(t)}{R_{i}}+\sum_{j=1}^{n} T_{i j} g_{j}\left(x_{j}\left(t-\tau_{j}(t)\right)\right)+\sum_{j=1}^{n} \sum_{l=1}^{n} T_{i j} g_{j}\left(x_{i}\left(t-\tau_{l}(t)\right)\right)+I_{i}, t \neq t_{k}, t \geq t_{0}, \\
& \Delta x_{i}(t)=d_{i} x_{i}\left(t^{-}\right), t=t_{k}, i=1,2, \ldots, n, k=1,2, \ldots,
\end{aligned}
$$

Where, $R_{i}(t)>0$ and $I_{i}(t)>0$ are the capacitance, the resistance, and the external input of the $i$ th neuron, respectively. $T_{i j}(t)>0, W_{i j}(t)>0$ and $T_{i j 1}(t)>0, W_{i j 1}(t)>0$ are the first- and second-order synaptic weights of the neural networks, respectively. $\tau_{i j}>0$ is the time delays, and satisfy $0<\tau_{j}(t)<\tau$ ( $\tau$ is a constant). $I_{i}(t)$ is the input of the unit $i .0 \leq t_{0}<\cdots<t_{k}<\cdots, t_{k} \rightarrow \infty$ for $k \rightarrow \infty$, and $x\left(t_{k}^{+}\right)=\lim _{s \rightarrow t^{+}} x(s), \quad x\left(t_{k}^{-}\right)=\lim _{s \rightarrow t^{-}} x(s)$. 
Let $x(t)=\left(x_{1}(t), x_{2}(t), \cdots, x_{n}(t)\right)^{T}$, and $\left.P C([-\tau, 0]), R^{n}\right)$ denote the set of piecewise right continuous functions $\phi:[-\tau, 0] \rightarrow R^{n}$ with the sup-norm $|\phi|_{t_{0}}=\sup _{t_{0}-\tau \leq s \leq t_{0}}\|\phi(s)\|$. For given $t_{0}$ and $\varphi \in P C\left([-\tau, 0], R^{n}\right)$, the initial condition of (1) is $x_{i}(s)=\varphi_{i}(s), s \in\left[t_{0}-\tau, t_{0}\right]$

In this paper, we assume the following conditions:

$$
\left|g_{i}(x)\right| \leq \sigma_{i}, 0 \leq \frac{g_{i}(x)-g_{i}(y)}{x-y} \leq K_{i}, \forall_{x}, y \in R, i=1,2, \ldots, n
$$

The lector $x^{*}=\left[x_{1}^{*}, \cdots, x_{n}^{*}\right]$ is the equilibrium point of system (1), if it is satisfies the following equation

$$
\frac{x_{i}^{*}}{R_{i}}=\sum_{j=1}^{n} T_{i j} g_{j}\left(x_{i}^{*}\left(t-\tau_{j}(t)\right)\right)+\sum_{j-1}^{n} \sum_{l=1}^{n} T_{i j l} g_{j}\left(x_{j}^{*}\left(t-\tau_{j}(t)\right)\right) g_{l}\left(x_{l}^{*}\left(t-\tau_{l}(t)\right)\right)+I_{i}, t \geq t_{0}, i=1,2, \ldots, n
$$

and suppose that some conditions are satisfied so that the equilibrium point of (1) does exist. Let $y_{i}(t)=x_{i}(t)-x_{i}^{*}, i=1,2, \cdots, n$ and $f_{i}\left(y_{i}\left(t-\tau_{i}(t)\right)=g_{i}\left(x_{i}\left(t-\tau_{i}(t)\right)-g_{i}\left(x_{i}^{*}\right)\right.\right.$. Then, for each $i=1, \cdots, n,\left|f_{i}(x)\right| \leq K_{i}(x), \quad x f_{i}(x) \geq 0, \quad \forall x \in R$

System (1) may be rewritten as follows

$$
\begin{aligned}
& C_{i} \frac{d y_{i}(t)}{d t}=-\frac{y_{i}(t)}{R_{i}}+\sum_{j=1}^{n}\left\{\mathrm{~T}_{i j}+\sum_{l=1}^{n}\left(T_{i j l}+T_{i l j}\right) \zeta_{l}\right\} g_{j}\left(y_{i}\left(t-\tau_{j}(t)\right)\right), t \neq t_{k}, t \geq t_{0} \\
& \Delta y_{i}(t)=d_{i} y_{i}\left(t^{-}\right), t=t_{k}, i=1,2, \ldots
\end{aligned}
$$

Where $\zeta_{l}$ is between $g_{l}\left(x_{l}\left(t-\tau_{l}(t)\right)\right)$ and $g_{l}\left(x_{l}^{*}\right)$.

As discussed in Section 1, in the real world, the neural network is often disturbed by environmental noises that affect the stability of the equilibrium point. In this paper, the model (5) is generalized as follows in order to account for the stochastic perturbations:

$$
\begin{aligned}
\frac{d y_{i}(t)}{d t} & =-\frac{y_{i}(t)}{C_{i} R_{i}}+\frac{1}{C} \sum_{j=1}^{n}\left\{T_{i j}+\sum_{l=1}^{n}\left(T_{i j l}+T_{i l j}\right) \zeta_{l}\right\} g_{j}\left(y_{i}\left(t-\tau_{j}(t)\right)\right)+\rho_{i}\left(t, y_{i}(t), y_{i}(t-\tau(t))\right) \dot{\omega}, t \neq t_{k}, t \geq t_{0}, \\
\Delta \mathrm{y}_{\mathrm{i}}(t) & =d_{i} y_{i}\left(t^{-}\right), \quad \mathrm{t}=\mathrm{t}_{\mathrm{k}}, \quad \mathrm{i}=1,2, \ldots \mathrm{n}, \mathrm{k}=1,2, \ldots
\end{aligned}
$$

The stochastic disturbance $\omega(t)=\left(\omega_{1}(t), \cdots, \omega_{n}(t)\right)^{T} \in R^{n}$ is a Brownian motion defined on a complete probability space $(\Omega, \Psi, P)$ with a natural filtration $\left\{\Psi_{t}\right\}_{t \geq 0}$ generated by $\{\omega(\mathrm{s}): 0 \leq \mathrm{s} \leq \mathrm{t}\}$, where we associate $\Omega$ with the canonical space generated by $\omega(t)$, and denote $\Psi$ the associated by $\{\omega(\mathrm{t})\}$ with the probability measure P. Assume that $\rho: R^{+} \times R^{n} \times R^{n} \rightarrow R^{n}$ is locally Lipschitz continuous and satisfies the linear growth condition.

$$
\begin{aligned}
& \operatorname{trace}\left[\rho^{\mathrm{T}}(\mathrm{t}, \mathrm{y}(\mathrm{t})), \mathrm{y}(\mathrm{t}-\tau(\mathrm{t})) \rho(\mathrm{t}, \mathrm{y}(\mathrm{t})), \mathrm{y}(\mathrm{t}-\tau(\mathrm{t}))\right] \leq\left|\mathrm{M}_{1} \mathrm{y}(\mathrm{t})\right|^{2}+\left|M_{2} y(t-\tau(t))\right|^{2} \\
& \text { Define } C=\operatorname{diag}\left(C_{1}, C_{2}, \ldots, C_{n}\right), \quad \mathrm{R}=\operatorname{diag}\left(\mathrm{R}_{1}, \mathrm{R}_{2}, \ldots, \mathrm{R}_{\mathrm{n}}\right) \\
& \mathrm{D}=\operatorname{diag}\left(\mathrm{d}_{1}, \mathrm{~d}_{2}, \ldots, \mathrm{d}_{\mathrm{n}}\right), \quad \mathrm{T}=\left(\mathrm{T}_{\mathrm{ij}}\right){ }_{\mathrm{n} \times \mathrm{n}}, \quad \mathrm{T}_{\mathrm{i}}\left(T_{i j l}\right)_{n \times n} \sum=\left(T_{1}+T_{1}^{T}, T_{1}+T_{1}^{T}, \ldots, T_{1}+T_{1}^{T}\right)^{T} \\
& f(y(t-\tau(t)))=\left[f_{1}\left(y_{1}\left(t-\tau_{1}(t)\right)\right), f_{2}\left(y_{2}\left(t-\tau_{2}(t)\right)\right), \ldots, f_{n}\left(y_{n}\left(t-\tau_{n}(t)\right)\right)\right]^{T} \\
& \zeta=\left|\zeta_{1}, \zeta_{2}, \ldots, \zeta_{n}\right|^{T}, \quad \Gamma=\operatorname{diag}(\zeta, \zeta, \ldots, \zeta) \\
& \Delta \mathrm{y}=\left[\Delta \mathrm{y}_{1}, \Delta \mathrm{y}_{2}, \ldots, \Delta \mathrm{y}_{\mathrm{n}}\right]^{\mathrm{T}}, \quad \mathrm{y}(\mathrm{t}-\tau(\mathrm{t}))=\left[\mathrm{y}_{1}\left(\mathrm{t}-\tau_{1}(\mathrm{t})\right), \ldots, \mathrm{y}_{\mathrm{n}}\left(\mathrm{t}-\tau_{\mathrm{n}}(\mathrm{t})\right)\right]^{\mathrm{T}}
\end{aligned}
$$

Then, we have 


$$
\begin{aligned}
& \left\{\begin{array}{l}
\frac{d y(t)}{d t}=-C^{-1} R^{-1} y(t)+C^{-1}\left(T+\Gamma^{T} \sum\right) f(y(t-\tau(t)))+\rho(t, y(t), y(t-\tau(t))) \dot{\omega}, t \neq t_{k}, t \geq t_{0}, \\
\Delta y(t)=D y\left(t^{-}\right), t=t_{k}, \quad i=1,2, \ldots, n, \quad \mathrm{k}=1,2, \ldots, \\
y(t)=\phi(t), t \in\left[t_{0}-\tau, t_{0}\right]
\end{array}\right. \\
& \phi(t)=\left[\phi_{1}(t), \phi_{2}(t), \ldots, \phi_{n}(t)\right]^{T}, \quad \phi(t)=\varphi(t)-x^{*}
\end{aligned}
$$

The following lemmas are useful in the proof of our results.

Lemma 1 (Schur complement) The following LMI

$\left[\begin{array}{ll}S(x) & Q(x) \\ Q^{T}(x) & P(x)\end{array}\right]<0$ is equivalent to $P(x)<0, Q(x) \mathrm{P}^{-1}(x) Q^{T}(x)<0$ where $S(x)=S^{T}(x)$, $P(x)=P^{T}(x)$ and $Q(x)$ depend on $x$.

Lemma 2 For any constant matrix $P=P^{T}>0$, scalar $0 \leq \tau(t) \leq \tau$, and vector function $\dot{x}:[-\tau, 0] \rightarrow R^{n}$ such that the following integration is well defined, then

$$
-\tau \int_{-\tau(t)}^{0} \dot{x}^{T}(t+s) P \dot{x}(t+s) d s \leq\left(x^{T}(t) \quad x^{T}(t-\tau(t))\left(\begin{array}{cc}
-P & P \\
P & -P
\end{array}\right)\left(\begin{array}{l}
x(t) \\
x(t-\tau(t))
\end{array}\right)\right.
$$

We are now in a position to introduce the notion of asymptotically stability for the impulsive stochastic neural networks (6) with time-delays.

Definition 1. System (6) is said to be asymptotically stable in mean square if for any given condition such that $\lim _{t \rightarrow \infty} E\|y(t)\|^{2}=0$, where $E\{$.$\} is the mathematical expectation.$

\section{Stability analysis}

In this section, new criteria are presented for the asymptotical stability of the equilibrium point of the impulsive stochastic neural networks (6) utilizing the Lyapunov-Krasovskii functional, and its results can be readily checked by using the Matlab LMI toolbox.

Theorem 1. The system (6) is asymptotically stable if there exist constants matrix $P \in R^{n \times n}, P=P^{T}>0, Q \in R^{n \times n}, Q=Q^{T}>0, L=\operatorname{diag}\left\{l_{1}, l_{2}, \cdots l_{n}\right\}>0$ and constants $\varepsilon>0, \rho>0$, such that $P \leq \rho I$,

$$
\begin{aligned}
& \Delta=\left[\begin{array}{cccc}
\Delta_{11} & e^{-\varepsilon \tau} Q & \Delta_{13} & \Delta_{14} \\
* & \Delta_{22} & L K & 0 \\
* & * & -2 L & \Delta_{34} \\
* & * & * & -Q
\end{array}\right]<0, \quad \Theta=\left[\begin{array}{cc}
-P & (I+D)^{T} P \\
P(I+D) & -P
\end{array}\right]<0, \\
& \Delta_{11}=\varepsilon P-R^{-1} C^{-1} P-P C^{-1} R^{-1}-e^{-\varepsilon \tau} Q+\rho M_{1}, \Delta_{22}=-e^{\varepsilon \tau} Q+\rho M_{2}, \Delta_{13}=P C^{-1}\left(T+T^{T} \Sigma\right),
\end{aligned}
$$

$\Delta_{14}=\tau R^{-1} C^{-1} Q, \Delta_{34}=\tau\left(T+T^{T} \sum\right)^{T} C^{-1} Q$ and $*$ denotes the symmetric terms in a symmetric matrix.

Proof. Choose the following Lyapunov-Krasovskii functional

$$
V(t)=e^{\varepsilon t} y^{T}(t) P y(t)+\int_{t-\tau}^{t} e^{\varepsilon s}(\tau-t+s) \dot{y}^{T}(s)(\tau Q) \dot{y}(s) d s \text { where } P=P^{T}>0 \text { and } Q=Q^{T}>0 \text { are }
$$
to be determined. Furthermore, there exists positive diagonal matrix $K=\operatorname{diag}\left(K_{1}, \cdots, K_{n}\right)$ such that the following inequality holds based on (4),

$$
\left.y^{T}(t-\tau(t)) L K f(y(t-\tau(t)))-f^{T}(y(t-\tau(t))) K f(t-\tau(t))\right) \geq 0,
$$

The weak infinitesimal operator $\ell$ of the stochastic process $\left\{\mathrm{y}_{t}=\mathrm{y}(\mathrm{t}+\mathrm{s}),-\tau \leq \mathrm{s} \leq 0\right\}$ is given. For $t \neq t_{k}(k=1,2, \cdots$,$) calculating the derivat -2 f^{T}(y(t-\tau(t)) L f(y(t-\tau(t))$ ives of $\mathrm{V}(\mathrm{t})$ along the trajectories of system (6) yields. 


$$
\begin{aligned}
& \ell V(t)=\varepsilon e^{\varepsilon t} y^{T}(t) P y(t)+2 e^{\varepsilon t} y^{T}(t) \mathrm{P} \dot{y}(t)+e^{\varepsilon t} \dot{y}^{T}(t)\left(\tau^{2} Q\right) \dot{y}(t)-\int_{t-\tau}^{t} e^{\varepsilon t} \dot{y}^{T}(\mathrm{~s})(\tau Q) \dot{y}(\mathrm{~s}) \mathrm{ds} \\
& \leq e^{\varepsilon t}\left[y^{T}(t)(\varepsilon P) y(t)+2 y^{T}(t) \mathrm{P} \dot{y}(t)+\dot{y}^{T}(t)\left(\tau^{2} Q\right) \dot{y}(t)-e^{-\varepsilon t} \int_{t-\tau}^{t} \dot{y}^{T}(\mathrm{~s})(\tau Q) \dot{y}(\mathrm{~s}) \mathrm{ds}\right. \\
& \leq e^{\varepsilon t}\left\{y^{T}(t)\left[\varepsilon P-R^{-1} C^{-1} P-P C^{-1} R^{-1}\right] y(t)+2 y^{T}(t) P C^{-1}\left(T+T^{T} \Sigma\right) f(y(t-\tau(t)))\right. \\
& \quad+\operatorname{trace}\left[\rho ^ { T } \left(t, y(t), y(t-\tau(t)) P \rho^{T}(t, y(t), y(t-\tau(t))]+\dot{y}^{T}(t)\left(\tau^{2} Q\right) \dot{y}(t)\right.\right. \\
& \quad-e^{\varepsilon \tau} \int_{t-\tau}^{t} \dot{y}^{T}(\mathrm{~s})(\tau Q) \dot{y}(\mathrm{~s}) \mathrm{ds}+2 f^{T}(y(t-\tau(t))) K L y(t-\tau(t)) \\
& \quad+\left[\mathrm { y } ^ { T } ( \mathrm { t } ) \mathrm { P } \rho \left(\mathrm{t}, \mathrm{y}(\mathrm{t}), \mathrm{y}(\mathrm{t}-\tau(\mathrm{t}))+\rho^{T}(\mathrm{t}, \mathrm{y}(\mathrm{t}), \mathrm{y}(\mathrm{t}-\tau(\mathrm{t}))] \mathrm{Py}(\mathrm{t}) \dot{w}\right.\right.
\end{aligned}
$$

We have $\ell V(t) \leq \mathrm{e}^{\varepsilon t}\left\{\eta^{T}(t) \Omega \eta(t)+\left[\mathrm{y}^{T}(t) \mathrm{P} \rho(\mathrm{t}, \mathrm{y}(\mathrm{t})), \mathrm{y}(\mathrm{t}-\tau(\mathrm{t}))+\rho^{T}(t, y(t), y(t-\tau(t))) P y(t)\right] w\right\}$ (9) Where $\Omega=\left[\begin{array}{lll}\Omega_{11} & e^{-\varepsilon \tau} Q & \Omega_{13} \\ * & \Omega_{22} & L K \\ * & * & \Omega_{33}\end{array}\right]$, with $\Omega_{11}=\varepsilon P-R^{-1} C^{-1} P-P C^{-1} R^{-1}+\rho M_{1}-e^{-\varepsilon \tau} Q$

$+R^{-1} C^{-1}\left(\tau^{2} Q\right) C^{-1} R^{-1}, \Omega_{22}=-e^{-\varepsilon \tau} Q+\rho M_{2}, \Omega_{13}=P C^{-1}\left(T+T^{T} \Sigma\right)-R^{-1} C^{-1}\left(\tau^{2} Q\right) C^{-1}\left(T+T^{T} \Sigma\right)$, $\Omega_{33}=-2 L+\left(T+T^{T} \sum\right)^{T} C^{-1}\left(\tau^{2} Q\right) C^{-1}\left(T+T^{T} \sum\right)$.form the conditions, we get $\Omega<0$.

Taking the mathematical expectation of both sides of (9), we obtain

$$
\frac{d E V(t)}{d t} \leq E\left(y^{T}(t) \Omega y(t)\right) \leq-\lambda_{\text {min }}(\Omega) E|y(t)|^{2}
$$

It means that the impulsive neural networks is asymptotically stable except possibly at the impulsive points $t_{k}(k=1,2 \cdots$,$) .To ensure the asymptotic stability, for t=t_{k}$, the following condition is required to be satisfied:

$$
V\left(t_{k}^{+}\right)-V\left(t_{k}\right)=y\left(t_{k}^{+}\right) P y\left(t_{k}^{+}\right)-y\left(t_{k}\right) P y\left(t_{k}\right) \leq \mathrm{e}^{\varepsilon t} y\left(t_{k}\right) P y\left(t_{k}\right)\left[(\mathrm{I}+\mathrm{D})^{T} P(I+D)\right] y\left(t_{k}\right)<0, k=1,2, \cdots
$$

This means that

$$
(I+D)^{T} P(I+D)-P<0 \text {. }
$$

From Lemma 1, we see that the inequality (10) is equivalent to that of (7). This completes the proof.

\section{Conclusions}

In this paper, we considered the delay-dependent stability criteria of impulsive stochastic Hopfield neural networks with time-varying delays. We use Lyapunov-Krasovskii Functional method and Linear Matrix Inequality (LMI) to deal with this problem. Some sufficient conditions are derived to ensure the asymptotical stability for the neural networks. Finally, numerical examples have demonstrated the effectiveness of the proposed results.

\section{References}

[1] J.J. Hopfield Neural networks and physical systems with emergent collective computational abilities, Proc. Natl. Acad. Sci., USA 79, 2554-2558. 1982.

[2] J.J. Hopfield Neurons with graded response have collective computational properties like those of two-state neurons, Proc. Natl. Acad. Sci., USA 81,3088-3092. 1984.

[3] G. Joya, M.A. Atencia and F. Sandoval Hopfield neural networks for optimization: study of the different dynamics, Neurocomputing, 43, 219-237. 2002 